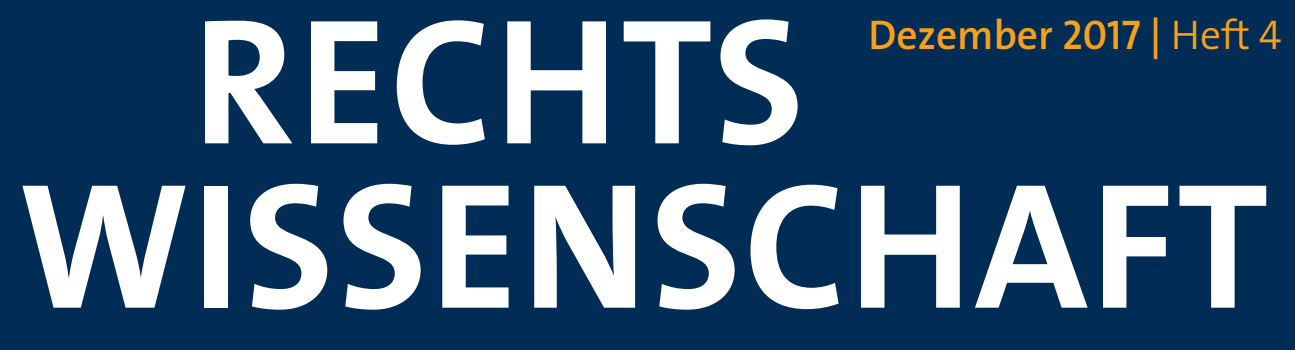

Zeitschrift für rechtswissenschaftliche Forschung

Herausgegeben von

Prof. Dr. Marietta Auer | Prof. Dr. Barbara Dauner-Lieb

Prof. Dr. Hans Christoph Grigoleit | Prof. Dr. Thomas Gutmann

Prof. Dr. Hans-Peter Haferkamp | Prof. Dr. Werner Heun $\dagger$

Prof. Dr. Tatjana Hörnle | Prof. Dr. Jens Kersten

Prof. Dr. Hans Kudlich | Prof. Dr. Frank Neubacher

Prof. Dr. Anne Peters | Prof. Dr. Thomas Pfeiffer

Prof. Dr. Helmut Satzger | Prof. Dr. Ewald Wiederin

Prof. Dr. Joachim Wieland

Aus dem Inhalt

\title{
Modernes Gewohnheitsrecht
}

Martin Klose

Die Bundesflagge ist schwarz-rot-gold

Rüdiger Zuck

„Fake News" und die „Lügenpresse“ - ein (neuer) Fall

für das Straf- und Ordnungswidrigkeitenrecht?

Frauke Rostalski

„New Directions in Law and Literature"-

Transatlantische Betrachtungen

Rico David Neugärtner 


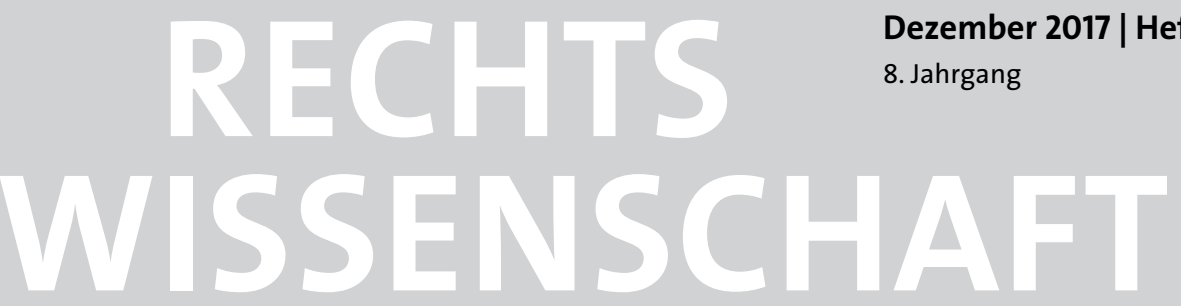

\section{Zeitschrift für rechtswissenschaftliche Forschung}

Herausgegeben von Prof. Dr. Marietta Auer M.A., LL.M., S.J.D. (Harvard), Giessen | Prof. Dr. Barbara Dauner-Lieb, Köln | Prof. Dr. Hans Christoph Grigoleit, München (LMU) | Prof. Dr. Thomas Gutmann, Münster | Prof. Dr. Hans-Peter Haferkamp, Köln | Prof. Dr. Dr. h.c. Werner Heun †, Göttingen | Prof. Dr. Tatjana Hörnle M.A. (Rutgers), Berlin (HU) | Prof. Dr. Jens Kersten, München (LMU) | Prof. Dr. Hans Kudlich, Erlangen | Prof. Dr. Frank Neubacher M.A., Köln | Prof. Dr. Anne Peters LL.M. (Harvard), Heidelberg/Basel | Prof. Dr. Dr. h.c. Thomas Pfeiffer, Heidelberg | Prof. Dr. Helmut Satzger, München (LMU) | Prof. Dr. Ewald Wiederin, Wien | Prof. Dr. Joachim Wieland LL.M., Speyer

Schriftleitung: Prof. Dr. Johannes Rux,Tübingen/Baden-Baden

\section{Inhaltsverzeichnis}

Johannes Rux

Danksagung.

\section{Abhandlungen}

Martin Klose

Modernes Gewohnheitsrecht

Rüdiger Zuck

Die Bundesflagge ist schwarz-rot-gold....

Frauke Rostalski

„Fake News“ und die „Lügenpresse“ - ein (neuer) Fall für das Straf- und

Ordnungswidrigkeitenrecht?

Rico David Neugärtner

„New Directions in Law and Literature“- Transatlantische Betrachtungen

Tagungen und ihre Folgen

Anne-Sophie Landwers/Marlene Voigt

TagungsberichtImmaterialgüter und Digitalisierung

Patrick Zurth

Blicke über den Tellerrand 


\section{Jahresregister}

Inhaltsverzeichnis nach Beitragsarten

Autorenverzeichnis

\section{Schriftleitung:}

Prof. Dr. Johannes Rux (V.i.S.d.P.) | Nomos Verlagsgesellschaft mbH \& Co. KG | Waldseestrasse 3-5 | D 76530 BadenBaden | Tel.: + 497221/210425 | Fax: + 497221/210427 | Mail: rux@nomos.de

Erscheinungsweise: 4 Ausgaben pro Jahr

Bezugspreise 2017: Jahresabonnement inkl. einem Onlinezugang Privatbezieher 179,- $€$, Institutionen (Mehrfachnutzung, unbegrenzte Anzahl an Online-Nutzern) 319,- $€$, Einzelheft 44,- $€$. Alle Preise verstehen sich incl. MWSt, zzgl. Vertriebskostenanteil.

Bestellmöglichkeit: Bestellungen beim örtlichen Buchhandel oder direkt bei der Nomos Verlagsgesellschaft BadenBaden

Kündigungsfrist: jeweils drei Monate vor Kalenderjahresende

Bankverbindung generell: Zahlungen jeweils im Voraus an Nomos Verlagsgesellschaft, Postbank Karlsruhe: DE07 660100750073636751 (IBAN), PBNKDEFF (BIC) oder Sparkasse Baden-Baden Gaggenau: DE05 66250030 0005002266 (IBAN), SOLADES1BAD (BIC)

Druck und Verlag: Nomos Verlagsgesellschaft mbH \& Co. KG | Waldseestrasse 3-5 | D-76530 Baden-Baden | Telefon (07221) 2104-o | Fax (07221) 2104-27| E-Mail: nomos@nomos.de

Anzeigen: Sales friendly Verlagsdienstleistungen | Pfaffenweg 15 | 53227 Bonn | Telefon (0228) 978980 | Fax (O228) 9789820 |E-Mail: roos@sales-friendly.de

Urheber- und Verlagsrechte: Die Zeitschrift sowie alle in ihr enthaltenen einzelnen Beiträge und Abbildungen sind urheberrechtlich geschützt. Jede Verwertung, die nicht ausdrücklich vom Urheberrechtsgesetz zugelassen ist, bedarf der vorherigen Zustimmung des Verlags. Mit der Annahme zur Veröffentlichung überträgt der Autor dem Verlag das ausschließliche Verlagsrecht für die Zeit bis zum Ablauf des Urheberrechts. Eingeschlossen sind insbesondere auch das Recht zur Herstellung elektronischer Versionen und zur Einspeicherung in Datenbanken sowie das Recht zu deren Vervielfältigung und Verbreitung online oder offline ohne zusätzliche Vergütung. Nach Ablauf eines Jahres kann der Autor anderen Verlagen eine einfache Abdruckgenehmigung erteilen; das Recht an der elektronischen Version verbleibt beim Verlag. Namentlich gekennzeichnete Beiträge geben nicht in jedem Fall die Meinung der Herausgeber/Redaktion oder des Verlages wieder. Unverlangt eingesendete Manuskripte - für die keine Haftung übernommen wird - gelten als Veröffentlichungsvorschlag zu den Bedingungen des Verlages. Die Redaktion behält sich eine längere Prüfungsfrist vor. Eine Haftung bei Beschädigung oder Verlust wird nicht übernommen. Bei unverlangt zugesandten Rezensionsstücken keine Garantie für Besprechung oder Rückgabe. Es werden nur unveröffentlichte Originalarbeiten angenommen. Die Verfasser erklären sich mit einer nicht sinnentstellenden redaktionellen Bearbeitung einverstanden.

Der Nomos Verlag beachtet die Regeln des Börsenvereins des Deutschen Buchhandels e.V. zur Verwendung von Buchrezensionen.

ISSN $1868-8098$ 